\title{
Soil-steel structure shell displacement functions based on tensometric measurements
}

https://doi.org/10.2478/sgem-2018-0020

received March 5, 2018; accepted June 18, 2018.

Abstract: This paper analyses the effects of loads that change their location, i.e. moving but quasi-static loads. Displacements defining the deformation of the soil-steel structure's shell buried in soil are calculated from the results of measurements performed using a dense grid of points located on the circumferential section of the corrugated plate. In this way, all the components of the structure, namely the corrugated plate, the backfill and the pavement with its foundation, as well as the natural (real) principles of their interaction, are taken into account in the solution. In the proposed algorithm, unit strains are converted into displacements, whereby results as accurate as the ones obtained by direct experimental measurements are obtained. The algorithm's main advantages are that the number of points is limitless, they are regularly distributed on the circumferential section of the shell and any displacement directions can be obtained. Consequently, the deformations of the shell can be faithfully reproduced. The algorithm's convenient feature is that one can use a simplified computational diagram of the shell in the form of a beam having the shape of the shell in 2D space (without the other components of the soil-steel structure). The advantage of this measuring method (electric resistance tensometry) is that there is no need to build the solid scaffold used for displacement measurements. The research focuses on the analysis of the displacements and the unit strains arising during the primary and secondary (return) travel of the load.

Keywords: soil-steel structure; moving loads; studies of shell deformations; displacement functions.

\footnotetext{
*Corresponding author: Czestaw Machelski, Faculty of Civil Engineering, Department of Bridges and Railways, Wroctaw University of Science and Technology, Wroctaw, Poland, E-mail: czeslaw.machelski@pwr.edu.pl
}

\section{Introduction}

The displacement-computing algorithm presented in this paper is based on the results of measurements of a bridge in Ostróda (Poland). A wildlife migration route and a local road pass under the bridge. There are four two-lane carriageways, separated by median strips, on the bridge. Hence, the width of the bridge is large, amounting to $B_{\mathrm{g}}=$ $61.82 \mathrm{~m}$ at the crest and to $B_{\mathrm{d}}=95.70 \mathrm{~m}$ at the embankment toe. This bridge structure holds the world record for shell span length [1] (the previous record belonged to a 24-m-long shell built in Canada).

The shells of corrugated-steel buried structures are made of metal plates. Using circular segments, one can make shells having complex cross-sectional shapes. The typical geometric feature of such shells is a circular plate (with radius of curvature $R$ ) in their top part (crown).The geometric characteristics of the shell's shape are its span length $L$ and height $H$, which for the considered bridge are given in Fig. 1. The geometry of the shell's corrugated plate is expressed by its technical designation UC $500 \times 237 \times 9.65$, standing for type UltraCor $a \times f \times t$, where $a$-corrugation length, $f$ - corrugation height and $t$ - plate thickness.

Shells made of corrugated plates are characterised by high stiffness [2], but only when buried in soil, i.e. when the soil-steel structures are put into service. As the thickness of the soil surcharge increases, the effect of live loads (a system of concentrated forces) decreases, as shown by the experimental results reported in this paper. Owing to road pavement or track superstructure rigidity also, the effects generated by vehicles are considerably reduced [2]. The range of the action of loads situated on the embankment, i.e. outside the shell, usually amounts to the shell's span length $L$.

Investigations of soil-steel structures under a load moving on the roadway usually involve dynamic testing, e.g. Bęben [3] and Mellat et al. [4]. Moreover, static testing plays an important role in determining the mechanics of structures such as shells sunk in the soil medium, as shown previously $[2,5-10]$. The influence functions used in bridge models, e.g. the study by Machelski [11], can 


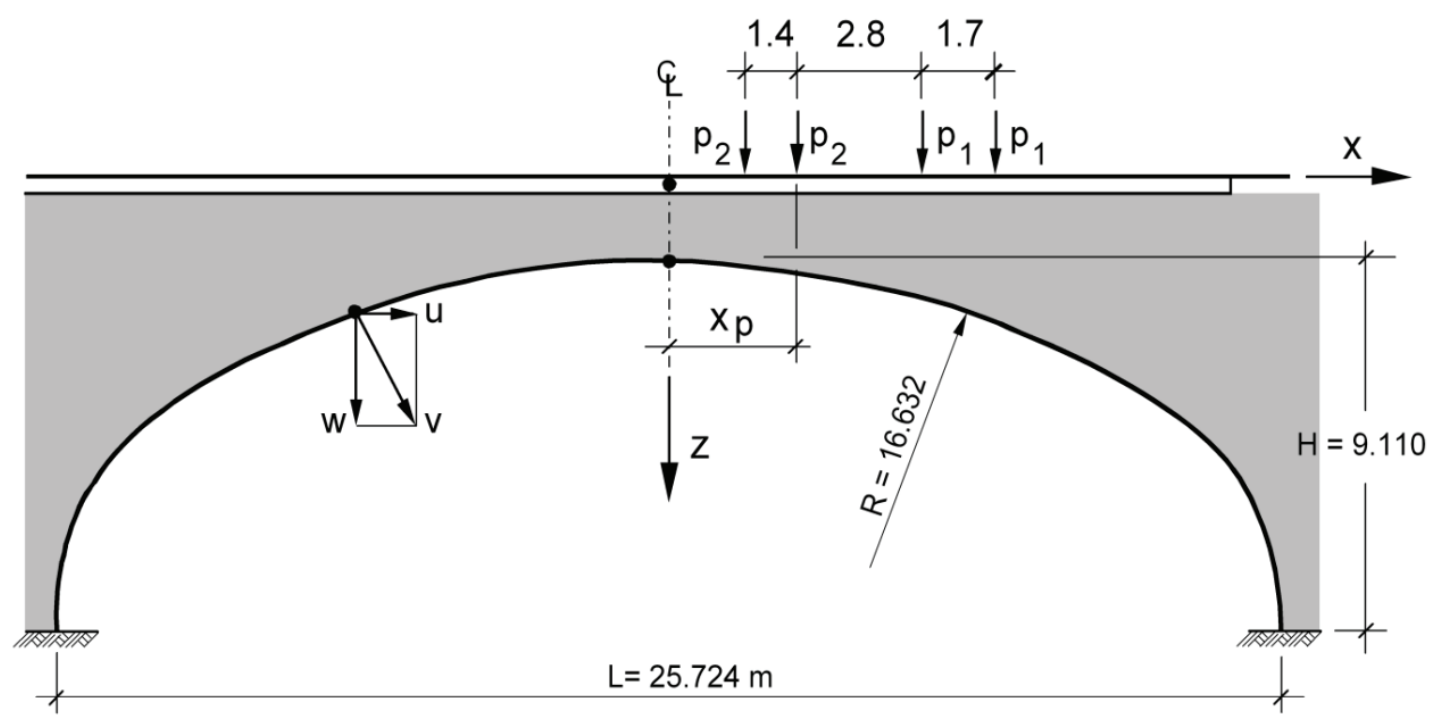

Figure 1: Cross-sectional geometry of soil-steel structure and truck location.

Table 1: Sequence of locations $x_{p}$ of the pair of trucks relative to shell crown.

\begin{tabular}{llllllll}
\hline$x_{\mathrm{p}} / L$ & $-4 / 4$ & $-3 / 4$ & $-2 / 4$ & $-1 / 4$ & 0 & $1 / 4$ & $2 / 4$ \\
\hline Primary travel & 0 & 1 & 2 & 3 & 4 & 5 & 6 \\
Secondary travel & - & 11 & 10 & 9 & 8 & 7 & 6 \\
\hline
\end{tabular}

be useful in such analyses. Influence functions can also be determined on the basis of measurements performed on soil-steel structures in service, subjected to a load in the form of a system of concentrated forces produced by vehicle wheels [2]. Then, the interaction of all the members, including the pavement (which, in typical bridges, is considered to be a secondary element not belonging to the structure), is taken into account.

The results of acceptance tests based on the concept of a moving load in the quasi-static sense are used in this paper. Two trucks driving parallel to each other along the road axis constituted the load. The trucks would drive stepwise, i.e. would stop at distances $x_{\mathrm{p}}$ (specified in Table 1) relative to the shell's crown - hence, the driving step is $L / 4$. Instrument readings would be taken at each of the stops. The load on each of the truck's front axles was $P_{1}=$ $60 \mathrm{kN}$ and that acting on each of the identical rear axles was $P_{2}=100 \mathrm{kN}$; hence, the truck's total weight $Q=32$ metric tons (altogether, 64 metric tons). In each of the measuring locations of the trucks during the travel there (the primary travel) and back (the secondary travel), the position of the wheels on the roadway was identical, as shown in Fig. 1. The trucks would drive without turning around. The full loading cycle lasted for nearly 3 hours, which gives an average time of about 12 min per measurement cycle. The loading cycle was based on the geodetic laser scanning of the deformations of the shell.

\section{Calculation of shell displacements}

A fragment of the soil-steel structure calculation model is shown in Fig. 2. An important component of such a structure is the pavement with its foundation. The material of this component and its physical properties are different from those of the backfill. Studies of corrugatedsteel buried structures have shown that the backfill greatly participates in carrying operational loads. The layered structure of the backfill is crucially important for the loadcarrying capacity of the soil-steel structure, determining the way in which the whole soil-steel structure deforms, as the shell deformation functions presented in this paper demonstrate. In this structural system, the soil-shell interface plays an important role.

Corrugated plates in soil-steel structure models are rendered as orthotropic shells or as a mesh-like beam structure [11]. The beam system facilitates computations in many ways, e.g. it becomes possible to formulate influence functions for the internal forces in the shell, which helps to determine the disadvantageous locations of loads on the bridge roadway. Such a representation of the corrugated plate was adopted in the following considerations. 


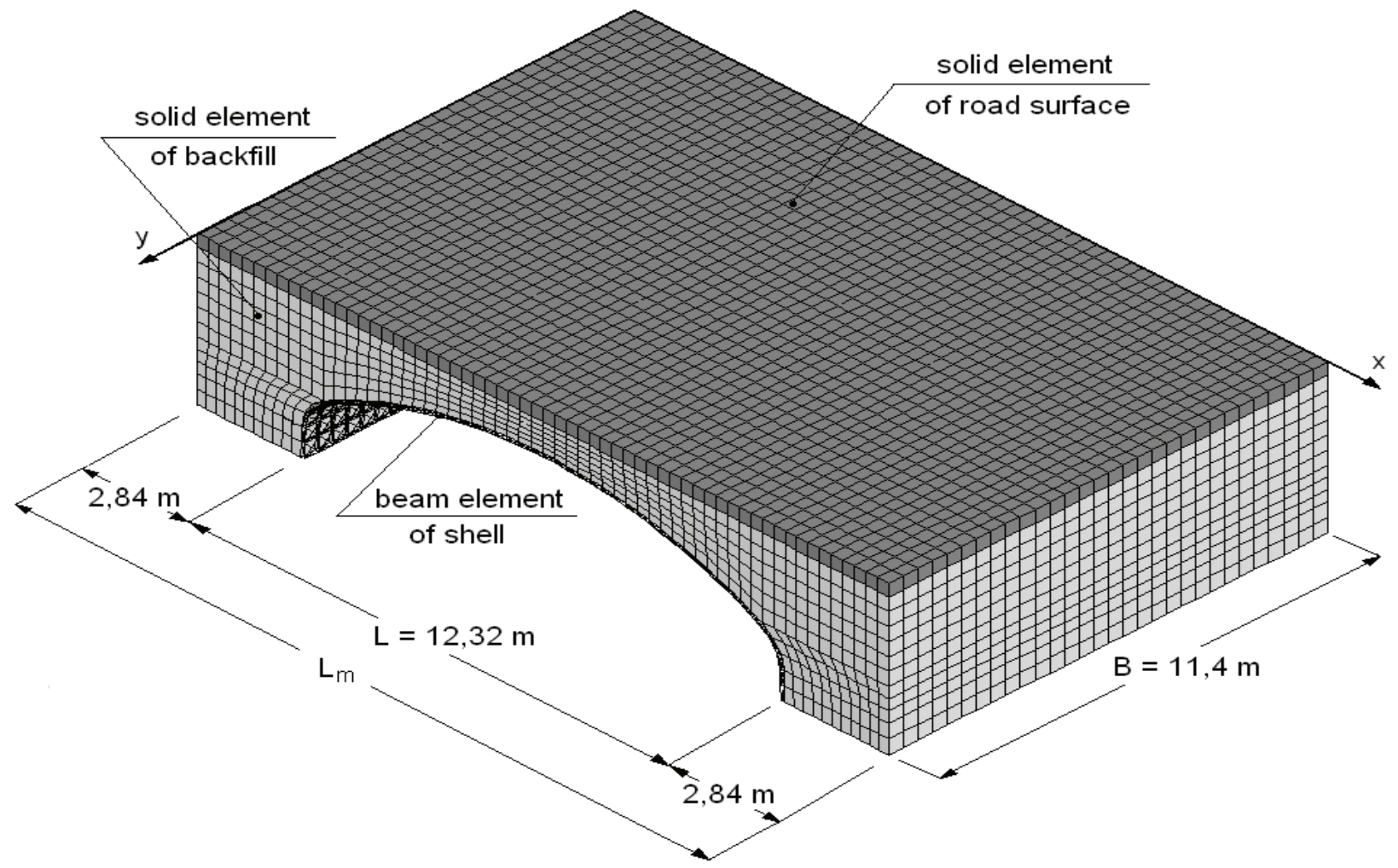

Figure 2: Model of soil-steel structure geometry [11].

In order to determine the displacement of any shell point in the radial direction $r$, one uses the classic integral algorithm as in the following equation for the beam system:

$$
r=\frac{1}{E I} \int M_{p} M_{r} d s+\frac{1}{E A} \int N_{p} N_{r} d s .
$$

Calculation result $r$ in Eq. (1) consists of two parts, originating from bending and compression, respectively. The quantities with subscript $p$ result from a vehicle load in the form of a system of concentrated forces $P$, as shown in Fig. 1. Internal forces $M_{\mathrm{p}}(\mathrm{s})$ and $N_{\mathrm{p}}(\mathrm{s})$ are functions related to the line of the shell's circumferential section, treated as the axis of a beam with an arch geometry, lying in plane $x z$. Functions $M_{\mathrm{p}}(\mathrm{s})$ and $N_{\mathrm{p}}(\mathrm{s})$ in Eq. (1) are considered to be the result of the solution of the structure model shown in Fig. 2, subjected to the system of forces $P$, which means that the functions were obtained in 3D geometry.

Integration is conducted over the whole length of the circumferential section, i.e. along an arc with length $s$ and, therefore, in planar system $x z$. Since the geometric parameters of this section as a beam are constant, the stiffnessare, respectively, $E I$ and $E A$, whereby they can be transposed to the front of the integral. The displacementtracking functions, denoted with subscript $r$, are internal forces $M_{\mathrm{r}}$ and $N_{\mathrm{r}}$, stemming from the unit load applied in the considered displacement point and in the radial direction. According to the reduction theorem used in structural mechanics, in this case, an equivalent model in the form of a planar arc with the geometry of the shell circumferential section, i.e. a $2 \mathrm{D}$ model, is used. The model is described further in this paper.

Obviously, the algorithm for calculating displacement $r$ from Eq. (1) in accordance with the procedure described earlier yields the same results as the direct calculation of $r$ in the model shown in Fig. 2, which means that it does not make much sense to use the displacement-computing algorithm since this involves considerable additional computational effort. However, this algorithm is effective when the results of in situ measurements, as in this paper, are used.

If unit strains in two points of the corrugation, as in the beam cross-section shown in Fig. 3, are to be calculated, two equations should be formulated as follows:

-one for the crest, i.e.

$$
\varepsilon_{g}=\frac{N_{p}}{E A}+\frac{M_{p}}{E I} v_{g},
$$


-and one for the valley, i.e.

$$
\varepsilon_{D}=\frac{N_{p}}{E A}-\frac{M_{p}}{E I} v_{D}
$$

From the difference between these values, one gets

$$
\varepsilon_{g}-\varepsilon_{D}=\frac{M_{p}}{E I} f .
$$

Equation (4) takes into account that $v_{g}+v_{D}=f$ is the distance between points $\mathbf{g}$ and $\mathbf{D}$ and, at the same time, it is the height of the corrugation. The value of $\varepsilon$, standing for the unit strain on the axis of inertia of the beam's crosssection, has been identified in Fig. 3. Hence, one gets the following relation:

$$
\varepsilon=\frac{N_{p}}{E A} .
$$

Therefore, Eq. (1) can be now presented in the following form:

$$
r=\int \frac{\varepsilon_{g}-\varepsilon_{D}}{f} M_{r} d s+\int \varepsilon \cdot N_{r} d s
$$

Equations (1) and (6) are identical, but for different load functions. The effects of external loads in Eq. (1) are expressed by internal forces, while in Eq. (6), they are expressed by unit strains. In both the solutions, the planar cross-section principle as applied to beam systems is used.

The advantage of the solution expressed by Eq. (6) is that the problem size is reduced to the shell's circumferential section isolated from the structure. The values of $\varepsilon_{\mathrm{g}}$ and $\varepsilon_{\mathrm{D}}$ contain complete information about the bridge's geometric structure (3D) and the shell support conditions, whereby the actual characteristics of the backfill material and the pavement material are specified. The distribution of the unit strains (function) along the length of the circumferential section takes into account the actual principles of the interaction between the structural members, e.g. the slip of the soil relative to the shell in the interfacial zone. Functions $\varepsilon_{\mathrm{g}}(\mathrm{s})$ and $\varepsilon_{\mathrm{D}}(\mathrm{s})$ reflect the changes in load location. Thus, the measured unit strains, identical as the directly measured displacement $r$, contain information about the actual deformation of the structure. It follows from the discussion that from Eq. (1), one can obtain a different value for the model than the one yielded by Eq. (6) for the actual structure. Of course, the result obtained from Eq. (6) is the accurate one since the model only approximately represents the geometry and physical properties of the structure.

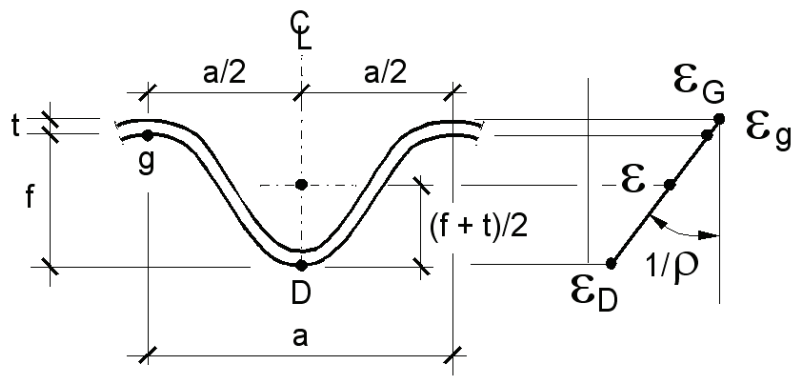

Figure 3: Cross-section of corrugated plate's circumferential section and distribution of unit strains.

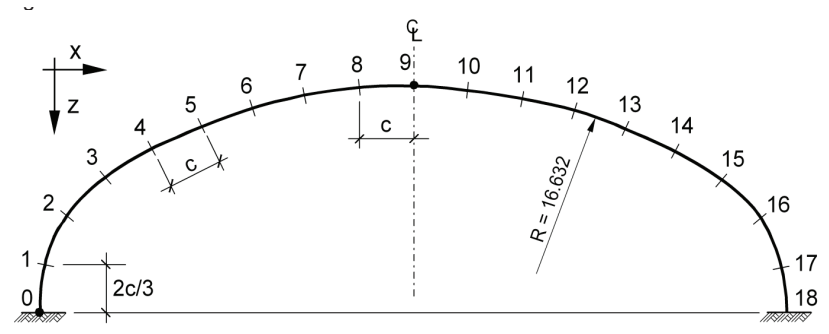

Figure 4: Arrangement of measuring points on the circumferential section of shell, as well as the $2 \mathrm{D}$ model.

\section{Results of tensometric measurements}

Unit strains in the soil-steel structure were measured using strain gauge sensors stuck on the corrugated plate's surface accessible from beneath. In Fig. 3, the results of the measurements are denoted as $\varepsilon_{\mathrm{g}}$ and $\varepsilon_{\mathrm{D}}$. The measuring points were regularly spaced in the circumferential direction of the shell band, as shown in Fig. 4. The measured unit strains $\varepsilon_{\mathrm{g}}$ and $\varepsilon_{\mathrm{D}}$ are used to determine the other characteristic deformations brought to a common line, as shown in Fig. 3. Hence, the deformation of the crest on the (inaccessible) soil backfill side is calculated as follows:

$$
\varepsilon_{G}=\frac{1}{f}\left[(f+t) \varepsilon_{g}-t \varepsilon_{D}\right] \mu \mathrm{m} / \mathrm{m} .
$$

Of primary importance is the value of the unit strain on the central axis, calculated from the following formula:

$$
\varepsilon=\frac{1}{2 f}\left[(f+t) \varepsilon_{g}+(f-t) \varepsilon_{D}\right] \mu \mathrm{m} / \mathrm{m}
$$

Figure 3 shows the geometric dependences (based on the planar cross-section principle) for unit strains in the cross-section of the circumferential section. It follows 

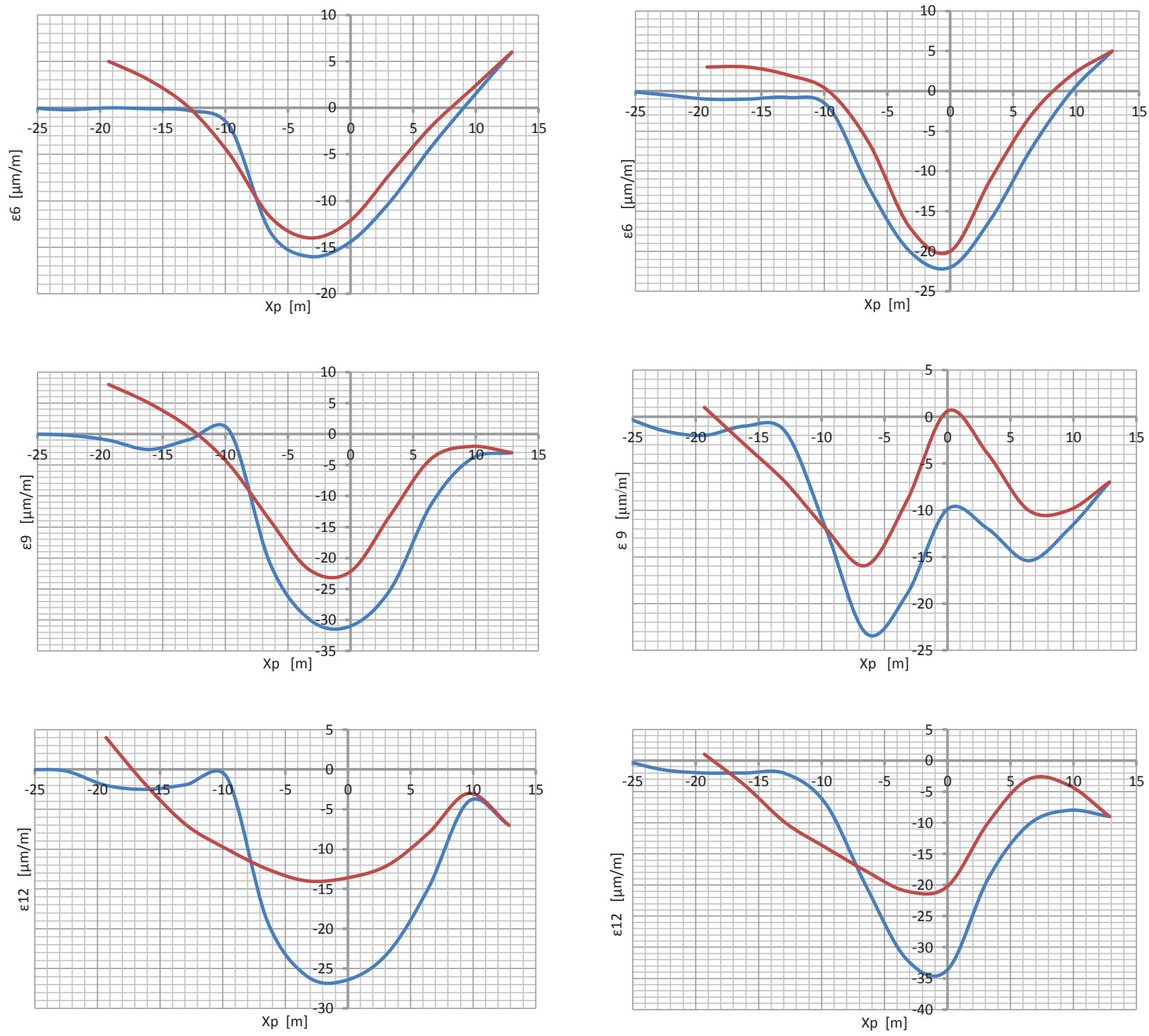

Figure 5: Functions $\varepsilon_{\mathrm{g}}\left(x_{\mathrm{p}}\right)$ for three points in the shell crown zone.

Figure 6: Functions $\varepsilon_{D}\left(x_{p}\right)$ for three points in the shell crown zone.

from them that the radius of curvature of beam $\rho$ and its curvature $\kappa$ are interrelated by the strength dependence given in the following formula:

$$
\kappa=\frac{1}{\rho}=\frac{\varepsilon_{g}-\varepsilon_{D}}{f},
$$

Figures 5 and 6 show the functions of the measured unit strains for the points selected from Fig. 4. Two points, namely 6 and 12, symmetrically situated relative to the shell crown 9, were selected. The $x$-axis shows the truck load locations relative to the shell crown, denoted with coordinate $x_{\mathrm{p}}$ in Fig. 1. Negative values (compression) predominate among the strain values in the diagrams, whereby the distributions are similar as in Fig. 3.

The shape of the graphs indicates that their form depends on the direction of vehicle travel: the primary travel and the secondary travel. The primary travel would start from the location of the vehicles beyond the structure $\left(x_{\mathrm{p}}=L\right)$, hence the zero reading. Then, the vehicles would drive forward, as shown in Fig. 2. When they reached the support location $\left(x_{p}=L / 2\right)$, the trucks would drive backwards (in reverse). The vehicles would be halted at the locations specified in Table 1 in order to record the results. This means that in any of the vehicle locations at a selected instant $x_{\mathrm{p}}$, the distribution of the forces generated by the vehicle axles is the same, as shown in Fig. 1 (no U-turn). A comparison of the graphs shown in Figs. 5 and 6 indicates that the shapes of the functions are different. The characteristic positive feature of the functions is that 
they approach zero when the measuring cycle is over, i.e. when $x_{\mathrm{p}}=-3 L / 4$, as in Table 1 .

The interdependences between $\varepsilon_{\mathrm{g}}\left(x_{\mathrm{p}}\right)$ and $\varepsilon_{\mathrm{D}}\left(x_{\mathrm{p}}\right)$ are not constant, but they can be similar. On the basis of the results presented in Figs. 5 and 6, one can formulate function $\varepsilon(x)$ as in Eq. (8). In the considered case, when $\varepsilon_{\mathrm{g}}$ and $\varepsilon_{\mathrm{D}}$ have the same sign (compression) and $t$ is many times lower than $f$, one can use the simplification $\varepsilon \approx\left(\varepsilon_{\mathrm{g}}+\right.$ $\left.\varepsilon_{\mathrm{D}}\right) / 2$. Hence, the relation $\varepsilon_{\mathrm{G}} \approx \varepsilon_{\mathrm{g}}$, calculated from Eq. (7), also holds. Such simplified dependences can be derived directly from Figs. 5 and 6.

Figure 7 shows functions $R \cdot k\left(x_{\mathrm{p}}\right)$, assuming the graph drawing principles given in Figs. 5 and 6. When presenting the results, it is convenient to relate the beam's curvature to its original (design) shape and to the value of $R$ as in Fig. 1. In this way, one gets the dimensionless curvature change index, shown in Eq. 10.

$$
R \cdot \kappa=\frac{R}{\rho}=\frac{R}{f}\left(\varepsilon_{g}-\varepsilon_{D}\right) \mu \mathrm{m} / \mathrm{m} .
$$

The shape of the graphs plotted for the three points indicates that they differ for both the primary and the secondary travel, when the points 6 and 12 are asymmetrically located also. The arrangement of the vehicle axles has little bearing on the lack of symmetry the vehicles are not positioned symmetrically relative to the shell crown during the primary and secondary travel (Fig. 1).

By comparing the effects of bending and compression, one can determine the eccentricity $(e)$ of the resultant force (axial force $N$ ) in a cross-section of the corrugated plate, using the following relation:

$$
e=\frac{I}{A \cdot f} \frac{\varepsilon_{g}-\varepsilon_{D}}{\varepsilon}=56.278 \frac{\varepsilon_{g}-\varepsilon_{D}}{\varepsilon}[\mathrm{mm}] .
$$

The graphs in Figs. 5 and 6 show slight deviations of the resultant form the of the cross-section core, considered to relate to $e$ in the strength of materials:

$$
z_{r}=\frac{2 I}{A(f+t)}=\frac{2 \cdot 96766}{14.51 \cdot 247}=54 \mathrm{~mm}
$$

Hence, the conclusion is reached that the compression should be qualified as compression with a small eccentricity.
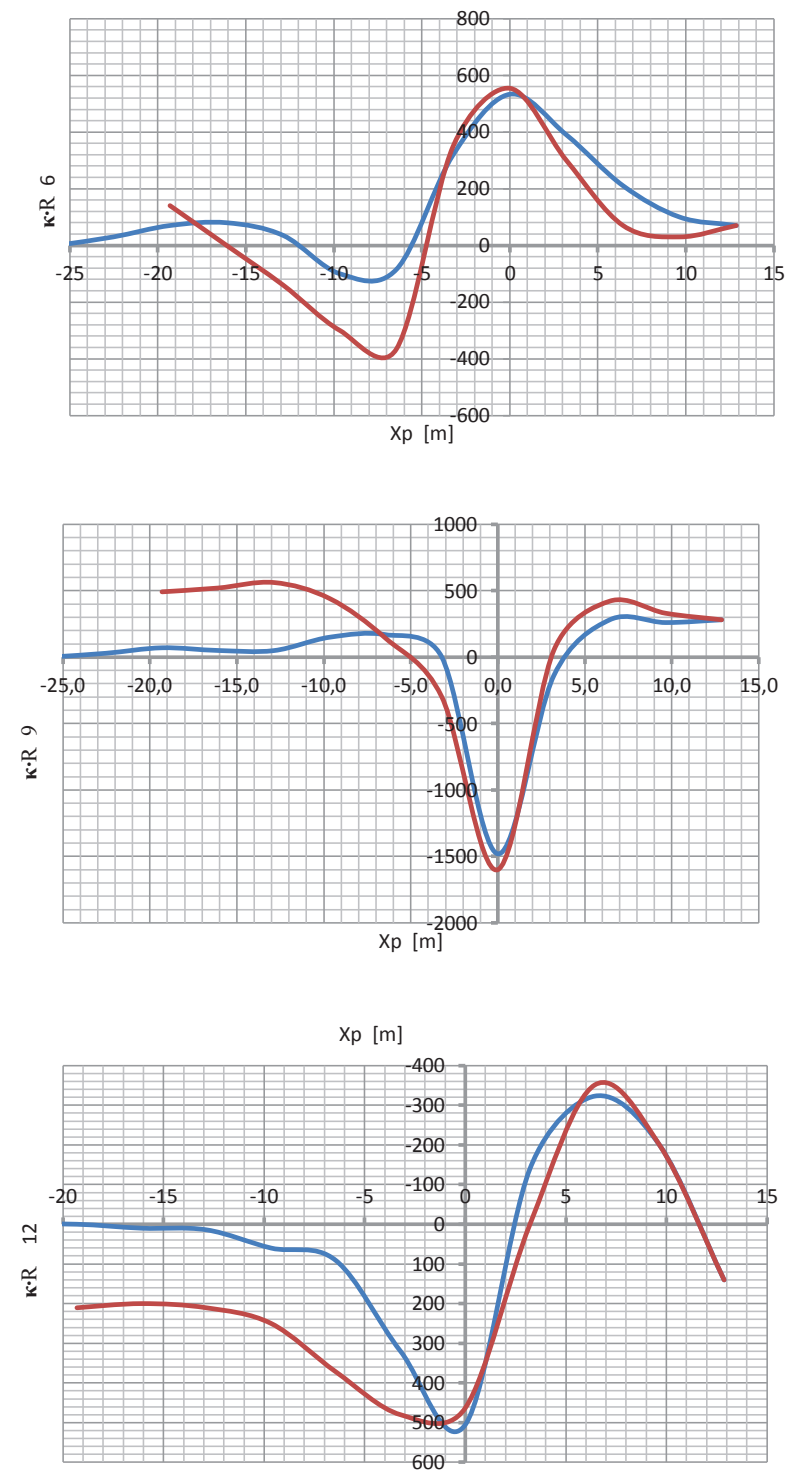

Figure 7: Functions $\rho\left(x_{p}\right)$ for three points in the shell crown zone, based on results presented in Figs. 5 and 6.

\section{Matrix algorithm for calculating displacements}

When the geometric relations in Eq. (9) for curvature change are introduced into Eq. (6), one gets the following equation:

$$
r=\int \kappa \cdot M_{r} d s+\int \varepsilon \cdot N_{r} d s
$$

Functions $\kappa(\mathrm{s})$ and $\varepsilon(\mathrm{s})$ are obtained as results of in situ measurements, and so these are in 3D geometry. Naturally, the quantities contain all the (earlier-mentioned) effects contributing to shell deformation. From Eq. (1), only 
the tracking functions $M_{\mathrm{r}}(\mathrm{s})$ and $N_{\mathrm{r}}(\mathrm{s})$, used to calculate displacement $r$, remain.

Because of the complex shapes of the functions in Eq. (13), instead of the integral form of the algorithm, it is convenient to use a matrix algorithm, as in the following equation:

$$
r=\frac{c}{6}\left(\boldsymbol{\kappa}^{T} \cdot \mathbf{B} \cdot \mathbf{m}_{r}+\boldsymbol{\varepsilon}^{T} \cdot \mathbf{B} \cdot \mathbf{n}_{r}\right) .
$$

In this approach, when the arc line is divided into several segments, each having length $c$ (as in Fig. 4), from the tracking functions in Eq. (13), one can form vectors $\mathbf{m}_{\mathrm{r}}$ and $\mathbf{n}_{\mathrm{r}}$ used in Eq. (14). These are vectors formed from the values of the functions in the arc points marked in Fig. 4. In the same way, the transposed vectors $\boldsymbol{\kappa}^{\mathrm{T}}$ and $\boldsymbol{\varepsilon}^{\mathrm{T}}$ are created from the load function.

Assuming that functions $\varepsilon(\mathrm{s})$ and $\kappa(\mathrm{s})$ are continuous, when continuous tracking functions $M_{\mathrm{r}}(\mathrm{s})$ and $N_{\mathrm{r}}(\mathrm{s})$ occur, one can use the band form of matrix B as in Eq. (15):

$$
\mathbf{B}=\left[\begin{array}{lllll}
\cdot & \cdot & & & \\
\cdot & 4 & 1 & & \\
& 1 & 4 & 1 & \\
& & 1 & 4 & . \\
& & & . & .
\end{array}\right] .
$$

When the function is discrete at the point of application of the concentrated force, as, e.g. in the case of $N_{\mathrm{r}}(\mathrm{s})$, one can adopt the division of matrix $\mathbf{B}$ into two blocks at this point:

$$
\mathbf{B}=\left[\begin{array}{llllll}
\cdot & \cdot & & & & \\
\cdot & 4 & 1 & & & \\
& 1 & 2 & & & \\
& & & 2 & 1 & \\
& & & 1 & 4 & \\
& & & & . & .
\end{array}\right] .
$$

When the displacement function $r\left(x_{\mathrm{p}}\right)$, represented by vector $\mathbf{r}$ in the matrix algorithm, is to be determined, it is advantageous to form the influence matrices $\mathbf{L}_{\mathrm{k}}$ and $\mathbf{L}_{\varepsilon}$ from vectors $\boldsymbol{\kappa}^{\mathrm{T}}$ and $\boldsymbol{\varepsilon}^{\mathrm{T}}$ and to use the relation in Eq. (17).

$$
\mathbf{r}=\frac{c}{6}\left(\mathbf{L}_{\kappa}{ }^{T} \cdot \mathbf{B} \cdot \mathbf{m}_{r}+\mathbf{L}_{\varepsilon}{ }^{T} \cdot \mathbf{B} \cdot \mathbf{n}_{r}\right) .
$$

\section{Comparative analyses of measuring techniques}

The changes in the shell crown deflection (point 9), derived as results yielded by different measuring techniques and denoted as T - tensometric, I - inductive and G - laser geodesy, are compared in Fig. 8. The deflection measured by the inductive sensors is regarded as the primary one, also because of its accuracy of $0.01 \mathrm{~mm}$. Graph I was obtained during the continuous two-way travel of the trucks (the additional measurement diagram). Considering the sensitivity of the strain gauges, the calculation results based on the tensometric measurements rank equally with the inductive sensor measurements (compare Fig. 5 and Fig. 6). The results yielded by the geodetic laser techniques are regarded as secondary since their accuracy is lower by one order, amounting to $0.1 \mathrm{~mm}$. However, due to the laser measurement, it was possible to determine displacements in the whole area of the circumferential section and not only at selected points as in the case of the $\mathrm{T}$ and I measurements.

The graphs for the primary travel presented in Fig. 8 are similar. The similarity between graphs $\mathrm{G}$ and I is natural in this case. In the case of inductive sensor measurements, a displacement is the result of the deformation of the whole circumferential section as a component of the analysed structure. In the case of tensometric measurements, deformations from the entire length of the shell's circumferential section are added up (integrated). Thus, when the number of points is sufficiently large and the displacement functions are quite regular, as in Figs.5 and 6 , the results are bound to agree. It should be noted that the tensometric and inductive measurement results agree for the entire travel of the trucks and not only for one location specified by $x_{\mathrm{p}}$. The agreement between graphs $\mathrm{T}$ and I also confirms the validity of the assumed strength principle of planar cross-sections in the investigated area of the corrugated plate, as in Fig. 3.

The result of displacement calculation, as in Eq. (14), consists of two factors stemming from bending and the axial force. Figure 9 shows the diagrams for the variation of the two factors obtained when calculating the deflection of point 6 of the shell. The proportions of the components vary greatly and depend also on the type of displacement ( $u$ or $w$ ) and the location of the measuring point. Such diagrams are useful when substantiating the peculiar shapes of the displacement functions presented further in this paper. 

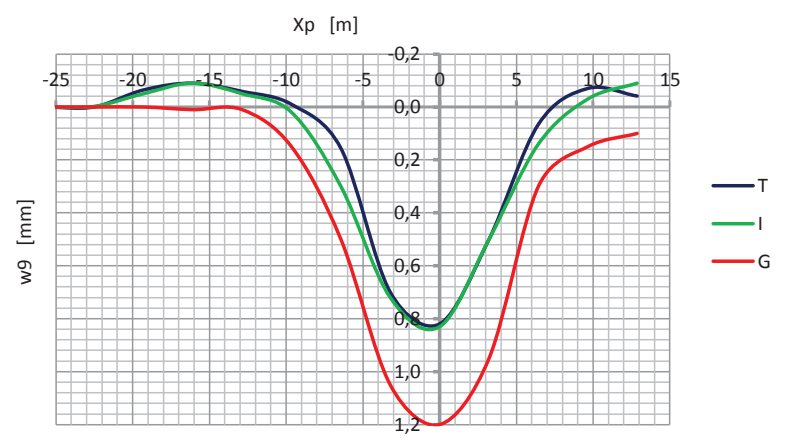

Figure 8: Comparison of shell crown deflections obtained by different measuring techniques.
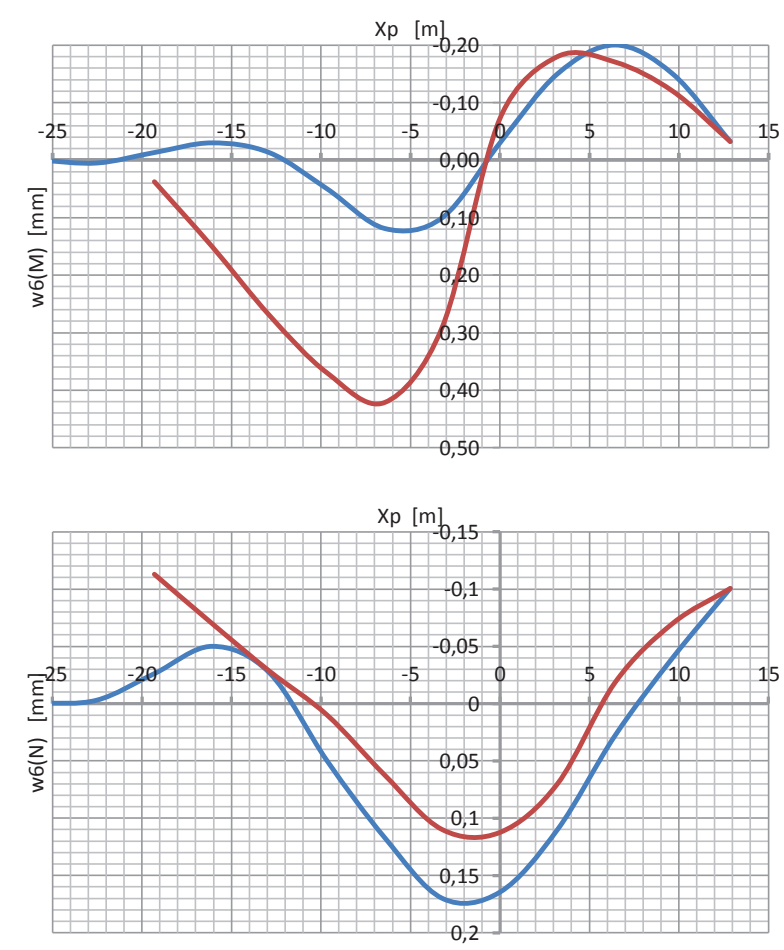

Figure 9: Deflection components at point 6 originating from bending and compression.

\section{Shell displacements based on tensometric measurements}

Figures 10 and 11 show selected examples of calculations using Eq. (17) and the results of tensometric measurements in the soil-steel structure's points given in Fig. 4. Using the general algorithm, one can calculate displacements at any selected shell points, in the form of components belonging to two groups: $r$ (radial) and $s$ (tangential); and $u$ (horizontal) and $w$ (vertical). On the basis of the two groups of displacement components, one can determine
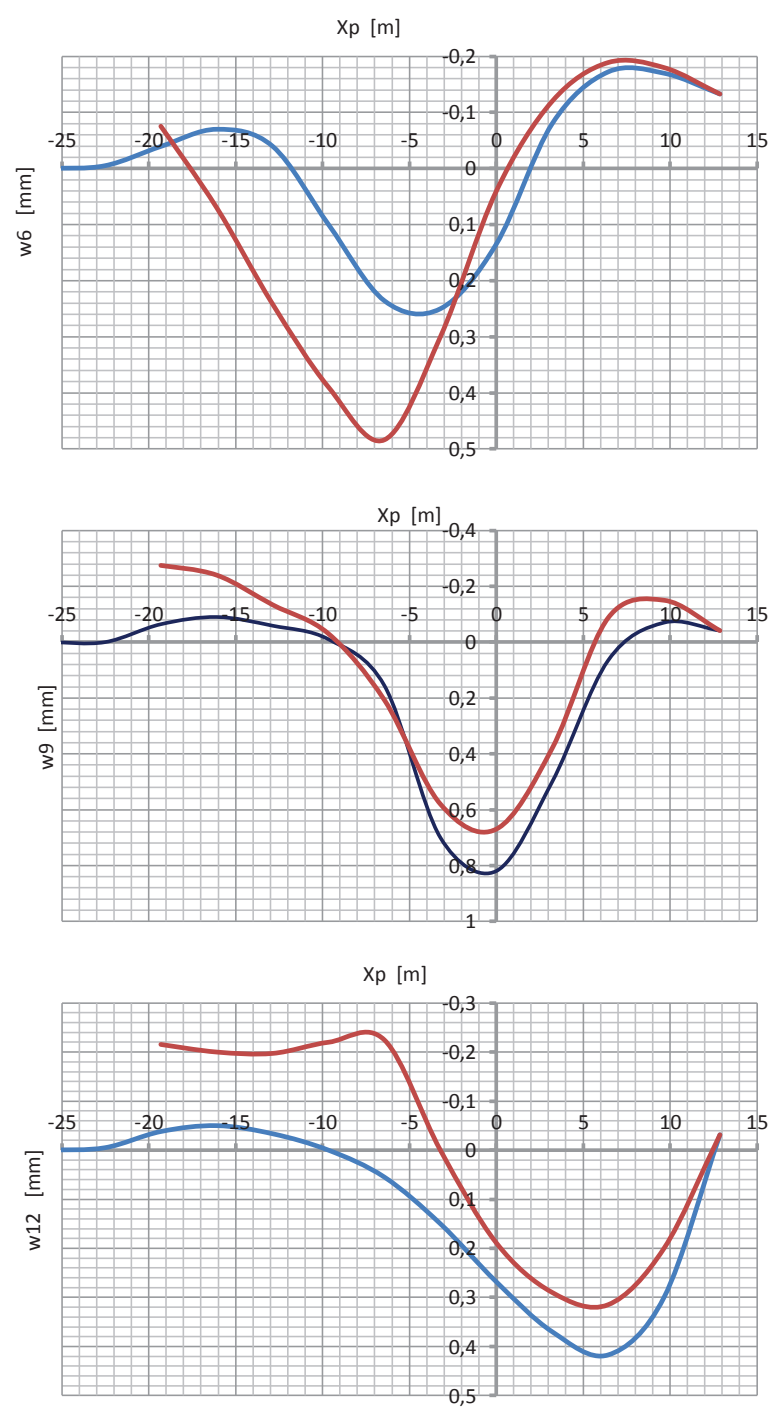

Figure 10: Changes in the vertical displacements of points in the shell crown area during the travel of vehicles.

the resultant displacement $v$ (Fig. 1) as the geometric sum, as follows:

$$
v=\sqrt{r^{2}+s^{2}}=\sqrt{w^{2}+u^{2}} .
$$

In the proposed algorithm, it is possible to select a point (not necessarily consistent with the configuration shown in Fig. 4) and a displacement direction. As a rule, the value of resultant $v$ is not determined since its direction is variable.

The positive characteristic of the behaviour of the soil-steel structure shell is that its deformation tends towards the initial state, i.e. towards the zero value of displacement after the full load cycle [7]. This happens in many situations (most often in the case of a shell crown deflection). Residual displacements often occur at points 


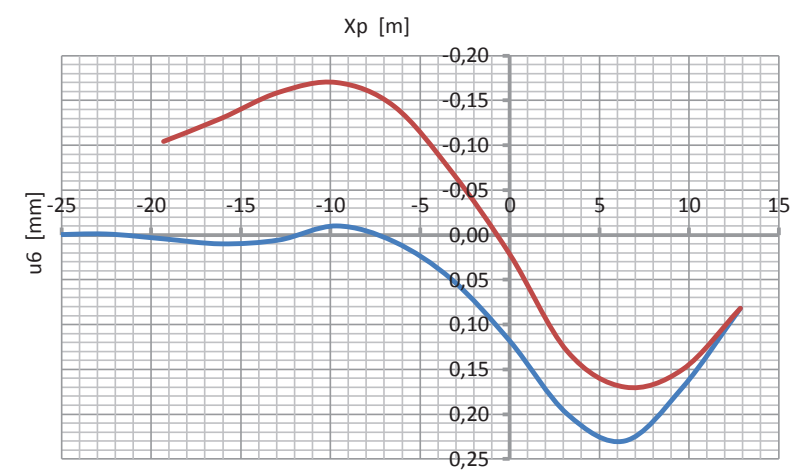

$X p[m]$

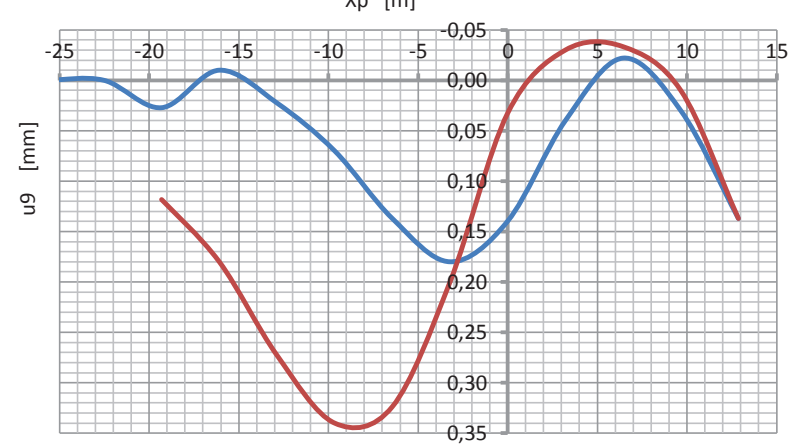

$\mathrm{Xp}[\mathrm{m}]$

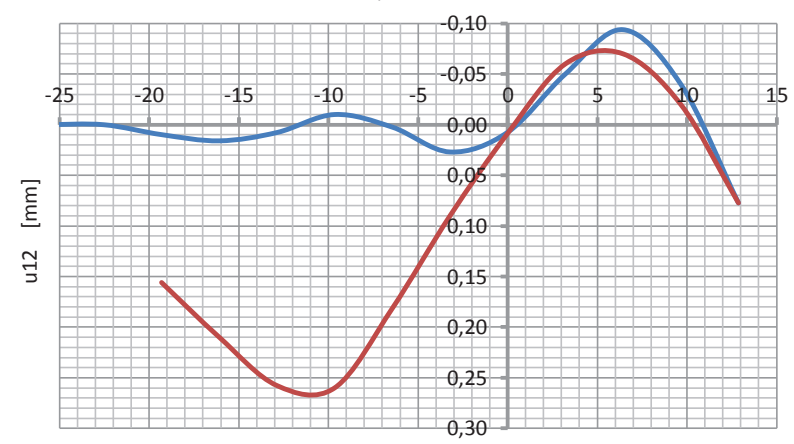

Figure 11: Changes in the horizontal displacements of three shell points during the travel of vehicles.

situated on the shell's side surface [5, 7]. Such a situation is shown in Figs 10, 11 and 12.

Figure 12 shows the shell deflection function $w(\tau)$ at point $x_{0}=\mathrm{L} / 4$, determined through continuous inductive measurement. The vehicles drove twice over the bridge at a speed of $\sim 2 \mathrm{~m} / \mathrm{sec}$. The maximum deflection occurred during the primary travel within time 0:09 $<\tau<1: 26$, and when the vehicles left the structure, the deflection decreased, but only to half of its original value. During the return (secondary) travel, when 2:44 $<\tau<3: 36$, a similar maximum deflection occurred again. No displacement reduction occurred after the full test cycle: the residual displacements remained. During the same test (but at a different point), the shell crown $\left(x_{0}=\mathrm{L} / 2\right)$ displacements

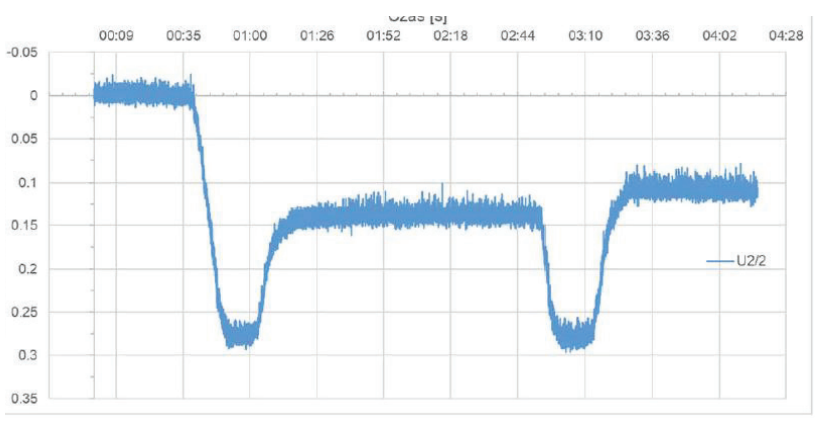

Figure 12: Change in vertical displacement of the shell point $x_{0}=L / 4$ during the travel of trucks.

were accompanied by trace residual effects. This phenomenon was analysed in earlier studies [5, 7], and it is referred to as a hysteresis loop in soil mechanics cyclic loading [12].

\section{Conclusion}

The effects of loads that change their location, i.e. moving loads, but with static characteristics, were analysed. The results of tensometric measurements performed for a dense grid of points located on the corrugated plate's circumferential section were used to calculate displacements characterising the deformation of a shell buried in soil. Consequently, the solution comprises all the soil-steel structure's members, namely the corrugated plate, the backfill and the pavement with its foundation, and it takes into account all the natural (real) principles governing the interactions between the members. This means that the structure's geometry and physical properties, as well as the load, are faithfully (accurately) reproduced in 3D. A convenient feature of the algorithm is that the functions of the component (directional) displacements $r, s, w$ and $u$ can be determined using a simplified shell - a beam having the shape of the shell's cross-section - and a 2D model (without the other structural members).

The proposed algorithm for converting unit strains into displacements yields accurate results. The advantage of this measuring method (electric resistance extensometry) is that there is no need for the solid scaffold used for displacement measurements. This can be useful when the access to the underside of the shell is difficult because of, for instance, boggy ground with a watercourse [8]. Another convenience is that any displacement direction can be selected. In order to directly measure displacements, one must properly fix (which poses difficulty) the sensor to the 
bent plate and build a stable measuring stand. The main advantage of the algorithm is the limitless number of points and their locations on the analysed circumferential section of the shell, as well as the arbitrary directions of displacements, whereby the deformations of the shell can be faithfully reproduced.

The results of the measurements show that the changes in unit strains differ between the primary travel and the secondary travel. This was observed previously when measuring the displacements of the shell in soilsteel structures [7]. The characteristic feature of the experimental results (unit strains) and the calculated geometric effects (e.g. $\rho$ ) and displacements is a hysteresis loop $[5,13]$. The problem of the behaviour of the backfillshell interface in such a hysteresis loop remains unsolved. Considering the two components of the solution in Eq. (17) and the diagrams shown in Fig. 9, one can notice that the deformation of the structure is due to the equivalent effects of bending and compression.

The algorithm requires a dense grid of measuring points. The form of the solution to be used when the number of measuring points is small has been presented in an earlier report [6]. In this case, a collocation algorithm for generating a 2D model (as in Fig. 1) is useful. Then, it is necessary to map the circumferential section of the whole structure, i.e. not only of the shell but also of the other members, namely the backfill and the pavement with the load, as shown in Fig. 2. Thus, in the solution described by Machelski and Janusz [6], the structure (3D geometry) is reduced to a planar (2D) model, as in Fig. 1.

\section{References}

[1] Machelski, C., Janusz, L., Tomala, P., Wiliams, K. (2018). Application of results of test in developing 2D model for soilsteel railway bridges. Conference Transportation Research Board of Nationals Academies, Washington D.C., 12-15 January 2018, Paper 19-05399.

[2] Machelski, C. (2015). Stiffness of railway soil-steel structures. Studia Geotechnica et Mechanica, 4, 29-36.

[3] Bęben, D. (2014). Experimental study on the dynamic impacts of service train loads on corrugated steel plate culvert. Journal of Bridge Engineering ASCE, 18(4), 339-346.

[4] Mellat, P., Anderson, A., Pettersson, L., Karuomi, R. (2014). Dynamic analysis of a short span soil-steel composite bridge for railways traffic using field measurements and numerical modelling. Engineering Structures, 69, 49-61.

[5] Sobótka, M. (2014). Numerical simulation of hysteretic live load effect in soil-steel bridge. Studia Geotechnica et Mechanica, 36.1, 103-109.

[6] Machelski, C., Janusz, L. (2017). Application of results of test in developing 2D model for soil-steel railway bridges.
Journal of the Transportation Research Board. Solid Mechanic. Transportation Research Board of Nationals Academies, Washington D.C., pp. 70-75.

[7] Machelski, C. (2014). Dependence of deformation of soil-shell structure on the direction of load passage. Roads and Bridges, 13, 223-233.

[8] White, K., Sargand, S., Massada, T. (2017). Evaluation of load rating procedure for metal culverts under shallow soil covers. Archives of Institute of Civil Engineering, 23, 311-323.

[9] Asp, O., Laaksonen, A. (2016). Instrumentation and FE-analysis of a large span culvert built under railway. Structural Engineering International, 26(4), 357-364.

[10] Moore, J.D., Regier, C., Hoult, N.A. (2017). Surface load testing of new circular and elliptical metal culverts at shallow cover. Archives of Institute of Civil Engineering, 23, 219-227.

[11] Machelski, C. (2010). Kinematic method for the determination of influence function of internal force in the steel shell of soilsteel structures. Studia Geotechnica et Mechanica, XXXII(3), 27-40.

[12] Sielver, M.L., Seed, H.B. (1971). Volume changes in sands during cyclic loading. ASCE Soil Mechanics and Foundations Division, 97(9), 1171-1182.

[13] Pettersson, L., Wadi, A., Williams, K. (2017). Structural design of flexible culverts developments trends. Archives of Institute of Civil Engineering, 23, 237-250. 